\title{
FuZZY SET THEORETIC APPROACH TO IMAGE THRESHOLDING
}

\author{
Ambar Dutta ${ }^{1}$, Avijit Kar $^{2}$ and B. N. Chatterji ${ }^{3}$ \\ ${ }^{1}$ Department of Computer Engineering, \\ Birla Institute of Technology, Mesra, Kolkata Campus, India \\ adutta@bitmesra.ac.in \\ ${ }^{2}$ Department of Computer Science and Engineering, \\ Jadavpur University, Kolkata, India \\ avijit_kar@cse.jdvu.ac.in \\ ${ }^{3}$ Department of Electronics and Communication Engineering, \\ B. P. Poddar Institute of Management and Technology, Kolkata, India \\ bnchatterji@gmail.com
}

\begin{abstract}
Thresholding is a fast, popular and computationally inexpensive segmentation technique that is always critical and decisive in some image processing applications. The result of image thresholding is not always satisfactory because of the presence of noise and vagueness and ambiguity among the classes. Since the theory of fuzzy sets is a generalization of the classical set theory, it has greater flexibility to capture faithfully the various aspects of incompleteness or imperfectness in information of situation. To overcome this problem, in this paper we proposed a two-stage fuzzy set theoretic approach to image thresholding utilizing the measure of fuzziness to evaluate the fuzziness of an image and to determine an adequate threshold value. At first, images are preprocessed to reduce noise without any loss of image details by fuzzy rule-based filtering and then in the final stage a suitable threshold is determined with the help of a fuzziness measure as a criterion function. Experimental results on test images have demonstrated the effectiveness of this method.
\end{abstract}

\section{KEYWORDS}

Fuzzy Sets, Thresholding, Measures of fuzziness, Noise Removal

\section{INTRODUCTION}

Image segmentation is that phase of image processing which partitions an image into a set of nonoverlapping meaningful homogeneous regions whose union is the entire image. Its objective is to extract the semantic objects lying in images either by dividing any given image into meaningful contiguous regions, or by extracting one or more important objects in images. The level upto which the partitioning is carried out depends on the problem being solved. There are various reasons for which segmentation is considered to be an extremely difficult task in image processing for non-trivial images: (1) lack of a priori knowledge about the number of segments present in the image; (2) variation of the gray scale values and their distributions from an image to another; and (3) inherent limitations of imaging sensors. Thus, vagueness in image processing arises from grayness ambiguity and uncertain knowledge about image. Uncertainty may arise at any stage in an image analysis system. Any decision made at a particular phase will have an

DOI : 10.5121/ijcsea.2011.1605 
impact on the subsequent phases. Hence, it is essential for an image analysis system to have sufficient provision for representing the uncertainties involved at every phase, so that the ultimate output of the system can be associated with the least uncertainties. One of the most popular tools for image segmentation is thresholding or gray level segmentation which consists of classification of the pixels as belonging to either the set of background pixels or the set of object pixels as a function of the intensity level. The threshold is determined by the histogram of the gray levels of pixels in the image. Thresholding is a transformation of an input image $g(x, y)$ into a segmented binary output image $b(x, y)$ as follow:

$$
b(x, y)= \begin{cases}b_{1} & \text { if } g(x, y)>T \\ b_{0} & \text { if } g(x, y) \leq T\end{cases}
$$

where the pixels having gray level intensity greater than $\mathrm{T}$ will belong to the object and are represented with a color $b_{1}$, while the pixels belonging to the background are represented with another color $b_{0}$.

The optimal choice of the threshold $\mathrm{T}^{*}$ of $\mathrm{T}$ based on a designed criterion function is a difficult task because of the presence of noise and vagueness and ambiguity among the classes. Recent advances in the fuzzy set theory provide the possibilities for developing new image segmentation techniques. Fuzzy models are capable to handle ambiguity and noise in the images and hence can improve the selection of optimal threshold for better image segmentation. Bezdek et al [1] classified fuzzy approaches for image segmentation into four categories: segmentation via thresholding, segmentation via clustering, supervised segmentation, supervised segmentation and rule-based segmentation. On the other hand, Tizhoosh [2] categorized fuzzy segmentation techniques into fuzzy thresholding, fuzzy rule, fuzzy clustering, fuzzy geometry and fuzzy integral based segmentation. Chaira and Ray [3] used fuzzy set theory to threshold an image and they introduced four types of fuzzy thresholding methods. Qiao et al [4] formulated a new criterion for segmenting small objects by exploring the knowledge about intensity contrast. Saha and Ray [5] introduced an adaptive thresholding technique via minimax optimization.

The remainder of this paper is structured as follows. Section 2 deals with basic related theories such as fuzzy set, membership function and several fuzziness measures those are useful for better understanding. We demonstrate our new fuzzy set-theoretic approach to threshold determination in section 3. In section 4, the superiority of our approach is illustrated with the help of experimental results. Finally, we conclude in section 5.

\section{RELATED THEORIES}

In this section, we explain a few relevant theories that will be helpful for further understanding and discussion.

\subsection{Fuzzy Set and Membership Function}

Fuzzy sets were introduced by Zadeh [6] in 1965 as a new way of representing vagueness in everyday life. Conventional sets contain objects that satisfy precise properties required for membership, whereas fuzzy sets contain objects that satisfy imprecisely defined properties to varying degrees. A fuzzy set $\mathrm{A}$ of the universe of disclosure $\mathrm{X}$ is defined as a collection of ordered pairs

$$
A=\left\{\left(\mu_{A}(x), x\right), \forall x \in X\right\}
$$


where $\mu_{A}(x)\left(0 \leq \mu_{A}(x) \leq 1\right)$ gives the degree of belonging of the element $\mathrm{x}$ to the set $\mathrm{A}$. The flexibility of fuzzy set theory is associated with the elasticity property of the concept of its membership function. The grade of membership is a measure of the compatibility of an object with the concept represented by a fuzzy set. Uncertainty in an image pattern may be explained in terms of grayness ambiguity or spatial ambiguity or both. Grayness ambiguity means 'indefiniteness' in deciding whether a pixel is white or black. Spatial ambiguity refers to 'indefiniteness' in the shape and geometry of a region within the image.

In order to threshold an image, it is essential to determine a membership function $\mu_{F}(f)$ associated with each gray level of the histogram. The membership function defines the degree of belonging of any pixel $f$ either to the background or the object set depending on the relationship between its gray level and the threshold $\mathrm{T}$. There are various ways in which a membership function can be defined. Irrespective of the measure of fuzziness used, an estimate of the mean gray level of the background $\mathrm{M}_{\mathrm{b}}$ and that of the objects $\mathrm{M}_{\mathrm{o}}$ is needed, where both values depend on the threshold T:

$$
\begin{gathered}
M_{b}(T)=\frac{\sum_{i=0}^{T} i * h(i)}{\sum_{i=0}^{T} h(i)} \\
M_{o}(T)=\frac{\sum_{i=T+1}^{L-1} i * h(i)}{\sum_{i=T+1}^{L-1} h(i)}
\end{gathered}
$$

The smaller the difference between the gray level of any pixel and the mean for its class, the greater will be the value of the membership function.

\subsection{Measures of Fuzziness}

There are two types of uncertainty: (1) vagueness that corresponds to the uncertainty associated with the problem of finding well defined borders between the objects to be segmented; and (2) ambiguity that corresponds to the uncertainty related to the difficulty of making the correct choice among two or more alternatives. The measure of fuzziness provides a way to measure the degree of fuzziness of a fuzzy set. A measure of fuzziness is a function

$$
I: F(X) \rightarrow \mathbb{R}^{+}
$$

where $F(X)$ denotes the set of all fuzzy subsets of $X$. There are few properties of the measures of fuzziness I:

$$
\begin{aligned}
& \text { 1. } I(A)=0 \text {, iff } \mu_{A}\left(x_{i}\right)=0 \text { or } 1, \forall i \\
& \text { 2. } I(A)=1 \text {, iff } \mu_{A}\left(x_{i}\right)=0.5, \forall i \\
& \text { 3. } I(A)=I(\bar{A})
\end{aligned}
$$

Below a few measures of fuzziness are discussed.

\subsubsection{De Luca and Termini's Entropy Measure of Fuzziness}

Motivated by Shannon's entropy measure [7], which is considered to be the fundamental base of the information theory, De Luca and Termini [8] proposed a non-probabilistic entropy function $\mathrm{f}(\mathrm{A})$ as a measure of fuzziness as follows. 
International Journal of Computer Science, Engineering and Applications (IJCSEA) Vol.1, No.6, December 2011

$$
\begin{gathered}
f(A)=\sum_{x \in X} S\left[\mu_{A}(x)\right], \text { where } \\
S\left[\mu_{A}(x)\right]=-\mu_{A}(x) \log _{2} \mu_{A}(x)-\left(1-\mu_{A}(x)\right) \log _{2}\left(1-\mu_{A}(x)\right)
\end{gathered}
$$

The normalized measure $\hat{f}(A)$ of $f(A)$ is defined as:

$$
\widehat{f}(A)=\frac{f(A)}{|X|} \quad(0 \leq \widehat{f}(A) \leq 1)
$$

where $\mathrm{IXI}$ is the cardinality of the universal set $\mathrm{X}$.

\subsubsection{Kauffmann's Measure of Fuzziness}

Kauffmann [9] proposed a measure of fuzziness as

$$
f(A)=\left\{\sum_{x \in X}\left|\mu_{A}(x)-\mu_{C}(x)\right|^{d}\right\}^{\frac{1}{d}}, d \geq 1
$$

where $\mu_{C}(x)$ is defined as

$$
\mu_{C}(x)= \begin{cases}0 & \text { if } \mu_{C}(x) \leq 0.5 \\ 1 & \text { if } \mu_{C}(x)>0.5\end{cases}
$$

\subsubsection{Yager's Measure of Fuzziness}

Yager [10] proposed a measure of fuzziness that depends on the relationship between the fuzzy set and its complement. The distance between a fuzzy set A and its complement is defined as:

$$
D_{d}(A, \bar{A})=\left[\sum_{i=1}^{n}\left|\mu_{A}\left(x_{i}\right)-\mu_{\bar{A}}\left(x_{i}\right)\right|^{d}\right]^{\frac{1}{d}}, i=1,2,3, \ldots, n
$$

The measure of fuzziness is defined as

$$
S(A)=1-\frac{D_{d}(A, \bar{A})}{|A|}
$$

\section{OUR APPROACH TO THRESHOLD DETERMINATION}

The thresholding scheme, proposed in this paper, works in two stages. In the first stage, images are preprocessed with the noise removal and edge preservation by fuzzy rule-based filtering. In the second and final stage, a suitable threshold is determined with the help of a fuzziness measure as a criterion function. Below we give a detailed description of the two stages.

\subsection{Preprocessing with Noise Removal}

The presence of noise affects the accuracy of image processing. In order to reduce the noise, nonlinear filtering techniques provide better result than linear filters as they do not degrade the edges and the details of the image. Recent advances in fuzzy logic gives new dimensions for developing new noise removal techniques. In this paper, in order to reduce noise we used a Gaussian fuzzy filter with mean center (GFFMC). The filters used in the image processing that employs fuzzy 
logic model are known as fuzzy filters. Let $x(i, j)$ be the input of a two-dimensional fuzzy filter, the output $y(i, j)$ of the fuzzy filter is defined as:

$$
y(i, j)=\frac{\sum_{(m, n) \in A} F[x(i+m, j+n)] \cdot x(i+m, j+n)}{\sum_{(m, n) \in A} F[x(i+m, j+n)]}
$$

where $F[x(i, j)]$ is the general window function and $A$ is the area of the window. For a square window of dimension $N \times N$, the range of $\mathrm{m}$ and $\mathrm{n}$ are: $-M \leq r, s \leq M$, where $\mathrm{N}=2 \mathrm{M}+1$.

Moreover, the Gaussian fuzzy filter with mean center (GFFMC) is defined as follows:

$$
F_{G F F M C}[x(i+m, j+n)]=e^{-\frac{1}{2} \frac{[x(i+m, j+n)-\bar{x}(i, j)]^{2}}{\sigma^{2}(i, j)}}
$$

where $\bar{x}(\mathrm{i}, \mathrm{j})$ and $\sigma(\mathrm{i}, \mathrm{j})$ denote the mean and standard deviation of all input values $\mathrm{x}(\mathrm{i}+\mathrm{m}, \mathrm{j}+$ $n$ ) respectively for $\mathrm{m}, \mathrm{n}$ in $\mathrm{A}$ at discrete indices $(\mathrm{i}, \mathrm{j})$. The reason for application of Gaussian fuzzy filter is to reduce both impulse noise and random noise from the image at the same time without degrading any image details.

\subsection{Determination of Threshold}

Huang and Wang [11] selected an optimal threshold by using the entropy measure as a criterion function. In this paper, we used an entropy-based fuzzy thresholding technique that uses the entropy of the background and foreground (object) regions, the entropy between the original and binarized image, relative entropy etc. The entropy of a random variable measures the uncertainty of the random variable and hence is a measure of the amount of information required to describe the random variable. Entropy serves as a measure of separation in an image that finds out the information of the two regions - above and below the threshold separately and calculates the entropy of the two classes. The entropies for all the gray levels below and above the threshold are added separately and then the entropy of the two classes is added to obtain the final entropy expression. The optimal threshold value is that gray level which corresponds to the maximum of all the calculated entropy expressions. Optimal threshold can also be obtained by minimizing the cross entropy of the two regions [12].

An L-level image $\mathrm{G}(M \times N)$ can be considered as an array of fuzzy singletons, where each singleton corresponds to an image pixel, each having a membership value denoting its degree of possessing some property (brightness, darkness etc). In the fuzzy set theoretic notation, it may be written as

$$
F=\left\{\mu_{F}(m, n): m=1,2, \ldots, M ; n=1,2, \ldots, N\right\}
$$

Then Shannon's entropy for an image of size $M \times N$ is given as

$$
H(F)=-\frac{1}{M N \ln 2} \sum_{i=0}^{M-1} \sum_{j=0}^{N-1}\left[\mu_{F}(i, j) \ln \left[\mu_{F}(i, j)\right]+\left[1-\mu_{F}(i, j)\right] \ln \left[1-\mu_{F}(i, j)\right]\right]
$$


International Journal of Computer Science, Engineering and Applications (IJCSEA) Vol.1, No.6, December 2011

which is used to obtain an optimum threshold. The entropy is calculated for each threshold gray level and the gray level that corresponds to the minimum entropy value represents the optimum threshold.

The normalized fuzziness function of an image can be calculated as:

$$
g(f, t)= \begin{cases}\sum_{f=0}^{L-1} h(f) S\left[\mu_{F}(f, t)\right] & \text { if } 0<\mu_{F}(f, t)<1 \text { for } 0 \leq t \leq L-1 \\ 0 & \text { if } \mu_{F}(f, t)=0 \text { or } \mu_{F}(f, t)=1\end{cases}
$$

where $h(f)$ represents the histogram of the image which shows the frequency distribution of the gray levels and

$$
\left.S\left[\mu_{F}(f, t)\right]=-\mu_{F}(f, t) \ln \left[\mu_{F}(f, t)\right]-\left[1-\mu_{F}(f, t)\right] \ln \left[1-\mu_{F}(f, t)\right]\right]
$$

Here in this paper, we have used Shannon's measure of fuzziness, but other fuzziness measures can also be used instead of it. The fuzziness function $g(f, t)$ is calculated for all the values of $t$. The optimal threshold $t^{*}$ is the gray level that minimizes $g(f, t)$ and hence it is the gray level that maximizes the separation of between the means of two classes as much as possible. Mathematically, it can be expressed as:

$$
t^{*}=\arg [\min g(f, t)] \quad 0 \leq t \leq L-1
$$

The detailed algorithm is given below:

1. Gaussian fuzzy filter is applied to reduce both impulse and random noise from the image at the same time.

2. The mean of the foreground and background regions are calculated for each threshold gray level.

3. The membership degree of the pixels of the image is calculated for each threshold gray level.

4. The fuzzy entropy is calculated for each threshold gray level.

5. The minimum value of the fuzzy threshold is selected.

6. The image is thresholded with the gray level corresponding to the minimum value of the fuzzy entropy. 


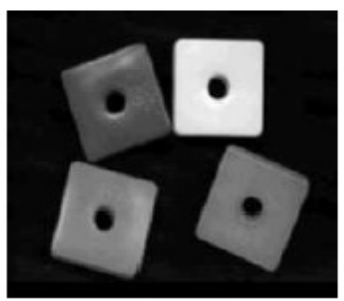

(a) Block

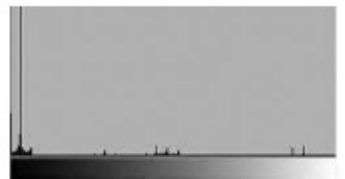

(d) Histogram of Block

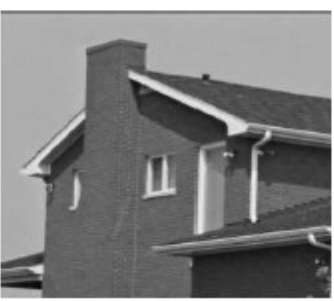

(b) House

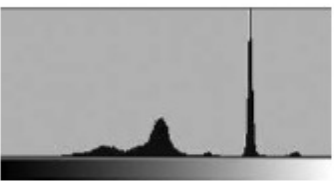

(e) Histogram of House

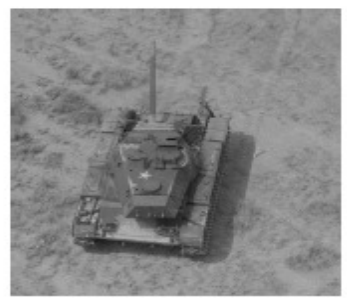

(c) Tank

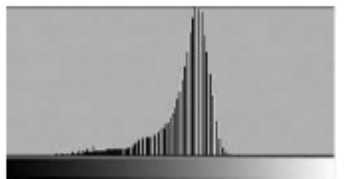

(f) Histogram of Tank

Figure 1: Test Images and their histograms

\section{EXPERIMENTAL RESULTS}

In order to evaluate the effectiveness of the proposed method, we have tested our algorithm with the help of a number of images, but due to limitation of space, in this paper we have shown the experimental results for only three test images given in Figure 1. Here, we have compared our algorithm with two well-known global thresholding techniques, proposed by Otsu [13] and Kittler-Illingworth [14] and one relatively new technique, proposed by Huang and Wang [11]. Otsu's method selected the threshold $t$ in such a way that the between-class variance is maximized and the intra-class variance is minimized. The algorithm positioned $t$ midway between the means of the two classes. On the other hand, Kittler and Illingworth assumed a Gaussian mixture model, that is, the pixels in the two categories came from a normal distribution and the gray level that minimized the number of misclassifications between the two normal distributions with the given means, variances, and proportions was considered as the threshold. Whereas, Huang and Wang proposed an algorithm for image thresholding by minimizing different measures of fuzziness.

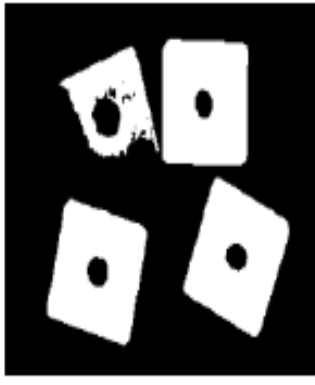

(a) Otsu

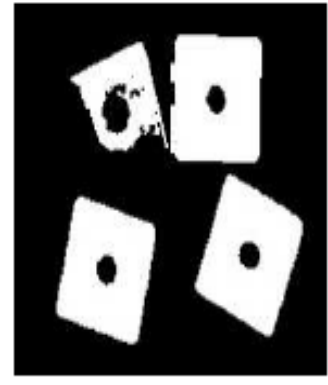

(b) Kittler-Illingworth

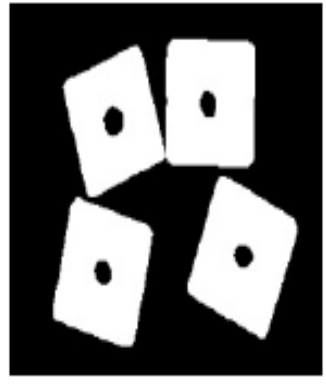

(c) Huang-Wang

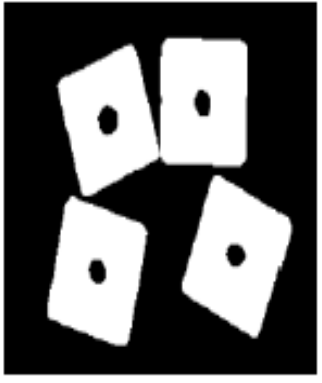

(d) Our Algorithm

Figure 2: Thresholding results for Block image 


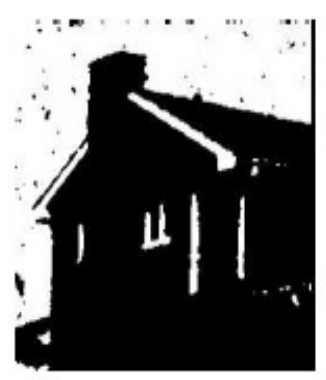

(a) Otsu

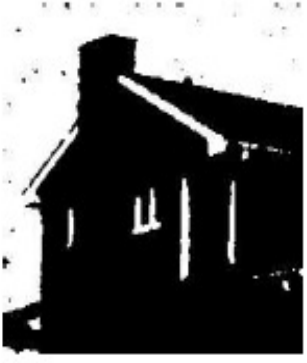

(b) Kittler-Illingworth

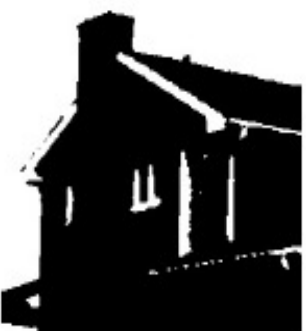

(c) Huang-Wang

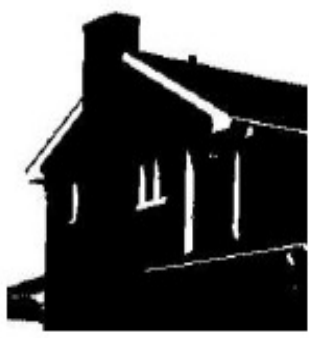

(d) Our Algorithm

Figure 3: Thresholding results for House image

It is observed from the figures Figure 2, Figure 3 and Figure 4 that our algorithm performs better than the two established thresholding techniques - Otsu's and Kittler-Illingworth's algorithms, and performs almost similarly (in fact, better for few test images) if we compare our algorithm with Huang-Wang's algorithm. In the presence of noise, our algorithm outperforms the three remaining algorithms considered in this paper.

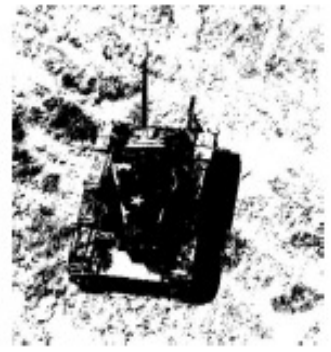

(a) Otsu

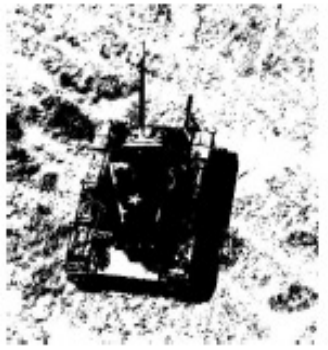

(b) Kittler-Illingworth

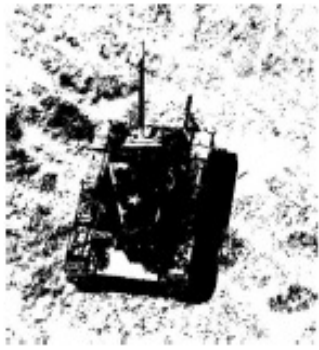

(c) Huang-Wang

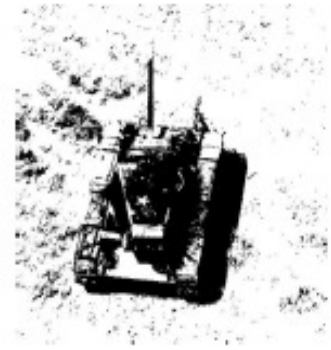

(d) Our Algorithm

Figure 4: Thresholding results for Tank image

Standard A4 (210mm x 297mm) portrait page set-up should be used. The left, right, top and bottom margins should be $30 \mathrm{~mm}$. Do not use any headers, footers or footnotes. No page numbers. Single column. All main text paragraphs, including the abstract, must be fully (left and right) justified. All text, including title, authors, headings, captions and body, will be Times New Roman font.

\section{CONCLUSIONS}

Image thresholding based on gray level histogram is an efficient and important tool for image segmentation. In this paper, we suggested a two-stage fuzzy set theoretic approach to image thresholding that uses the measure of fuzziness to evaluate the fuzziness of an image and to find an optimal threshold value. Fuzzy based methods consider the uncertainty in the image due to the imprecise pixel gray levels and vagueness in the image regions and boundaries which is incorporated in the form of membership function. Initially, images are preprocessed to reduce noise components without affecting much image details by fuzzy rule-based filtering and then in the second phase, a suitable threshold is determined with the help of a fuzziness measure as a 
International Journal of Computer Science, Engineering and Applications (IJCSEA) Vol.1, No.6, December 2011

criterion function. We demonstrated the effectiveness of our algorithm with the help of a number of test images. We shall try to extend this work to color images.

\section{REFERENCES}

[1] J. C. Bezdek, J. Keller, K. Raghu and N. R. Pal, Fuzzy models and algorithms for pattern recognition and image processing, The Handbooks of Fuzzy Sets Series, Kluwer Academic Publishers, Boston/London/Dordrecht, 1999.

[2] H. R. Tizhoosh, Image thresholding using type II fuzzy sets, Pattern Recognition, Vol. 38, No. 12, $2363-2372,2005$.

[3] T. Chaira and A. K. Ray, Threshold selection using fuzzy set theory, Pattern Recognition Letters, Vol. 25, No. 8, 865 - 874, 2004.

[4] Y. Qiao, Q. Hu, G. Qian, S. Luo and W. L. Nowinski, Thresholding based on variance and intensity contrast, Pattern Recognition, Vol. 40, Iss. 2, 596 - 608, 2007

[5] B. N. Saha and N. Ray, Image thresholding by variational minimax optimization, Pattern Recognition, Vol. 42, Iss. 5, 843 - 856, 2009.

[6] L. A. Zadeh, Fuzzy Sets, Information Control, Vol. 8, 338 - 353, 1965

[7] C. E. Shannon, A Mathematical Theory of Communication, Bell System Technical Journal, Vol. 27, $379-423,1948$

[8] A. De Luca and S. Termini, A definition of a non-probabilistic entropy in the setting of fuzzy set theory, Information and Control, Vol. 20, 301 - 312, 1972.

[9] Kaufmann, Introduction to the Theory of Fuzzy Subsets - Fundamental Theoretical Elements, Vol. 1, Academic Press, New York, 1975.

[10] R. R. Yager, On the measure of fuzziness and negation, Part - 1: Membership in the unit interval, International Journal of General Systems, Vol. 5, 221 - 229, 1979.

[11] L.K. Huang and M.J. Wang, Image thresholding by minimizing the measure of fuzziness, Pattern Recognition, Vol. 28, No. 1, 41 - 51, 1995.

[12] C.H. Li and C.K. Lee, Minimum cross entropy thresholding, Pattern Recognition, Vol. 26, No. 4, 617 $-625,1993$.

[13] N. Otsu, A threshold selection method from gray-level histogram, IEEE Transactions on Systems, Man and Cybernetics, Vol. 9, No. 1, 62 - 66, 1979.

[14] J. Kittler and J. Illingworth, Minimum error thresholding, Pattern Recognition, Vol. 19, No. 1, 41 47, 1986.

\section{Authors}

Ambar Dutta did his B.Sc. (Honors) in Mathematics from Presidency College, Kolkata in 1999 and MCA from Jadavpur University, Kolkata in 2002. He is working as a Lecturer in the department of Computer Science and Engineering, Birla Institute of Technology, Mesra, Kolkata Extension Centre. He s ubmitted his PhD thesis in December, 2010 in Jadavpur University, Kolkata in the area of image processing (corner detection and matching). His research interest includes Image Processing, Pattern Recognition, Data Mining and Soft Computing.

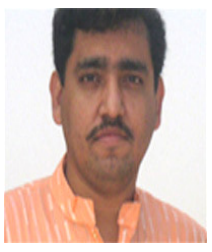


International Journal of Computer Science, Engineering and Applications (IJCSEA) Vol.1, No.6, December 2011

Avijit Kar did his M.Sc. and Ph.D in 1980 and 1984 respectively from IIT Kharagpur. $\mathrm{He}$ is currently a professor in the department of Computer Science and Engineering in Jadavpur University, Kolkata. He has supervised several $\mathrm{PhD}$ theses and is actively involved in many R \& D activities and IT related consultancy for Government of India and the private sector. His research interest includes biomedical imaging as well as SAR imaging. He is also into computer systems reliability. He is involved in a large number of industry sponsored development projects.

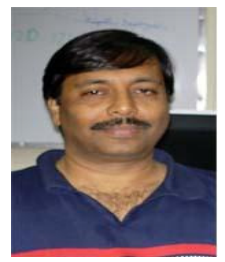

B. N. Chatterji obtained BTech (Hons) (1965) and Phd (1970) in Electronics and Electrical Communication Engineering of IIT, Kharagpur. He did Post Doctoral work at University of Erlangen-Nurenberg, Germany during 1972 - 73. Worked with Telerad Pvt Ltd, Bombay (1965), Central Electronics Research Institute, Pilani (1966) and IIT, Kharagpur as faculty member during 1967 - 2005. He was Professor during $1980-$ 2005, Head of the Department during 1987 - 1991, Dean Academic Affairs during 1994 - 1997 and Member of Board of Governors of IIT, Kharagpur during 1998 - 2000. He has published about 150 journal papers, 200 conference papers and four books. He was Chairman of four International Conferences and ten National Conferences. He has coordinated 25 short-term courses and was the chief investigator of 24 Sponsored Projects. He is the Fellow/Life Member/Member of eight Professional Societies. He has received ten National Awards on the basis of his Academic/Research contributions. His areas of interests are Pattern Recognition, Image Processing, Signal Processing, Parallel Processing and Control Systems. 